\title{
Research on Emotional Interaction Problems and Countermeasures of Intelligent Products based on Consumer Behaviours Analysis
}

\author{
Ning Yang ${ }^{1 *}$ \\ ${ }^{1}$ Jinan University, Jinan University - University of Birmingham Joint Institute, Information and computing science \& \\ Mathematics and Applied Mathematics, China \\ * Ning Yang: yangning2018050481@stu2018.jnu.edu.cn
}

\begin{abstract}
Intelligent products should not only pay attention to their mechanical function, but also give users emotional and spiritual pleasure, have higher personification degree and improve the use experience. Intelligent products should interact with users naturally, perceive the different moods, environments and mood changes of users, find the core needs of users through intelligent technology, and provide suggestions and solutions quickly and accurately. By using the methods of literature research, investigation and comparative analysis, this paper discusses the emotional interaction design scheme of intelligent products based on consumer behavior. Through a questionnaire survey, focus group forum and analysis of collected data, the paper summarizes the influence of emotional interaction mode of intelligent products and the expression mode and voice tone of an intelligent voice assistant on user experience, purchase standard and product personification degree, and the satisfaction and hope improvement of consumers when using intelligent products. In addition, the personalized design of product shape, color and scene mode should be carried out according to the different user groups, so as to produce an intelligent product with higher user comfort and dependence, promote the development of intelligent products and improve user experience.
\end{abstract}

Keywords: Intelligent products, Emotional Interaction Problems, Consumer Behaviours Analysis.

\section{INTRODUCTION}

With the rapid development of the Internet and intelligent technology, the process of information technology is accelerating. More products are developing in the direction of intelligence. People's dependence on machines is gradually increasing. For example, the washing machine which can automatically and intelligently identify the color and quantity of clothing fabrics to determine the amount of water and washing liquid; the sweeping robot which can avoid obstacles intelligently, and combine dry and wet cleaning; Smart bracelet, a wearable intelligent product, can monitor our heartbeat, blood pressure and other data at any time, and then give real-time exercise suggestions; intelligent products related to children's learning can make personalized learning plans according to the user's performance fluctuation and learning situation. All of them bring great benefits to people's life. At the same time, the vigorous development of e-commerce platforms and high-tech companies has also brought huge economic benefits. The change of lifestyle and shopping style is quietly changing our society, followed by the transformation and development opportunities of all walks of life. The gradual optimization of intelligent products has reduced the employment and development costs of many enterprises and companies, but also increased many new jobs to solve social employment problems. Only by combining science and technology with people's feelings, can we have a better development market, be accepted by more consumers, and bring huge economic benefits and social development. The ultimate goal of science and technology is to enable everyone to enjoy the welfare of science and technology development and facilitate people's life.

With the rapid development of all kinds of science and technology products, it is very important to update the products that meet the needs of customers. Users are no longer satisfied with the cold machine and its various simple functions, but gradually pay more attention to 
the use experience and emotional and spiritual pleasure. Even the appearance of the product, the voice of the voice system, and different scenarios in different environments all affect the user's ratings to varying degrees. In 1997, Picard of MIT put forward the concept of affective computing. By giving machines the ability to recognize, understand and express human emotions, computers have higher intelligence. The relationship between people and intelligent products is not only a relationship of asking for and executing orders, but also a role that intelligent products can communicate with like friends. When users use it, it not only completes a task, but also shares happiness with people after that. It requires designers to design an intelligent product based on user, scene and emotional mode. By combing the relevant literature, Liao Qinglin found that the higher the degree of emotional interaction, the higher the degree of personification of product perception. Under the same conditions, the degree of personification of intelligent voice assistant of female image is higher than that of intelligent voice assistant of male image. The degree of personification of product perception and user satisfaction is in line with the linear regression model, that is, to a certain extent, with the increase of personification, the degree of user satisfaction will increase. It should be increased. Users hope that the smart home assistant can initiate dialogue actively, and record user habits and basic information appropriately, so as to realize multiple rounds of dialogue and continuous interaction [1]. Norman once said, "We know a lot of information products, especially those closely related to our daily life. The design goal of the products is not only to make products more reasonable, more efficient, more multifunctional and more operational tools, but also to become a more comfortable and interesting emotional design, which has gradually become an important factor for users to buy the product." [2] Lin Wenqian used a convolution neural network to collect a variety of human physiological parameters through wearable devices, and tried to send different control commands to the manipulator according to the change of emotion [3].

Most scholars mainly study the application of emotional interaction design in intelligent products or emotional computing, but there is still a lack of research on emotional interaction of intelligent products based on consumer behaviour analysis. This paper will use the literature research method, survey method and comparative analysis method to find out the shortcomings of current intelligent products and the future development and improvement aspects. Through the feedback analysis of users after use, we found some problems, such as the response of intelligent products when calling is too indifferent; the intelligent bracelet cannot give encouragement when running for new mileage; the appearance design of children's intelligent wearable products is too common or the emergency call positioning function is not perfect and so on. We will sort out and count these problems, sort out some of the most popular imperfect functions, analyse the status quo and find solutions. Finally, design intelligent products with higher user experience and satisfaction under the support of artificial intelligence technology.

\section{METHODOLOGY}

The ultimate goal of emotional interaction is to make the computer automatically adjust on the premise of understanding human emotional state, so that users can have a more perfect experience when using products and rely on intelligent products. In order to achieve this goal, we analyzed a lot of user feedback and some sales data, and carried out comparative analysis and in-depth research, using three methods: literature research method, survey method and comparative analysis method.

\subsection{Literature research method}

Literature review, also known as research review, refers to the paper formed by analysing, summarizing, sorting out and commenting on the existing research results and problems of an academic problem (or research field) in a certain period of time on the basis of comprehensively mastering and analysing the relevant literature of an academic problem (or research field). Literature review generally needs to objectively describe and comment on the current research situation, so as to predict the development and research trend or seek new research breakthrough. The writing steps of literature review can be generally divided into literature collection, literature reading and classification, literature processing, comparison and review, forecasting trends or proposing problems to be further studied [4]. Through reading journals and papers, this paper understands that there are still insufficient researches on emotional interaction of intelligent products at present; through the interpretation of intelligent product industry report, this paper deeply explores the development status and prospects of intelligent products; through reading relevant books, this paper understands the concept and characteristics of emotional interaction

\subsection{Survey method}

Investigation and research method refers to the research method of obtaining relevant materials directly through investigation and understanding of the objective situation, and analysing these materials. In this paper, through the analysis of user reviews of intelligent products, we will find out the functions that users most want to update, and design diversified and personalized product function adjustments that can 
attract customers, so as to maximize the economic benefits.

\subsection{Comparative analysis}

Comparative analysis method is an analysis method that compares two or more things or objects to find out the similarities and differences between them [5]. This paper will compare the user feedback and product sales changes before and after the same product upgrade, judge whether the economic benefits brought by the function upgrade meet the expectations, and make further product upgrade plan and user satisfaction survey.

\section{RESULT}

\subsection{Survey and Analysis on emotional interaction of intelligent products (based on survey) (questionnaire)}

Ghost s et al. from Samsung company found that for mobile phone voice assistants, calm, professional and rational characters are more favoured by users, and the personality and emotion of virtual assistants should not be displayed too much [6]. Niculescu et al. conducted a group of experiments on Hotel inquiry robots to study the influence of tone, humour and compassion of social robots on user experience [7]. Lee et al. believe that for smart home products, emotion and companionship are the key influencing factors of user satisfaction [8]. Therefore, based on the study and thinking of the above conclusions, we conducted a questionnaire survey and data collection and analysis of group focus Forum on the emotional interaction of intelligent products.

\subsubsection{The role of intelligent products}

Through the data analysis of the questionnaire survey, we found that respondents are more willing to treat intelligent products as family and friends in life, so product design should pay more attention to emotional accompaniment, communication and other functions, so that users can trust and rely on intelligent products.

\section{Roles}

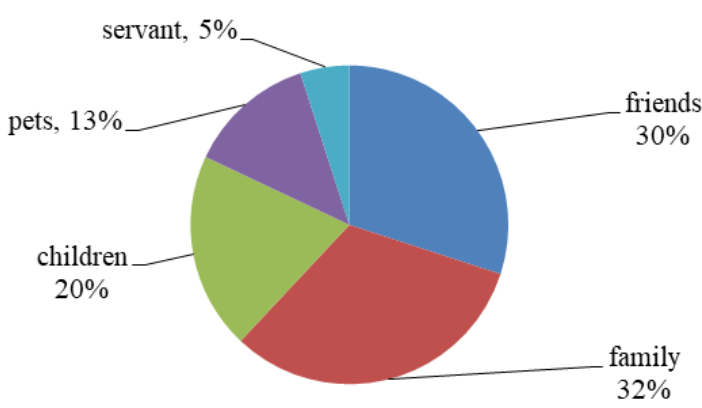

Fig.1 The role of intelligent products

\subsubsection{The basis of judging product role}

Respondents' judgment on the role of smart appliances in your life mainly comes from the expression and voice tone of smart products. It shows that intelligent products need to be constantly updated and strengthened in terms of personification to bring users the best experience. So that users can give smart appliances the most needed role in their mind, and truly meet the needs of users. At the same time, it makes a variety of modes of voice intonation and response time setting function, so that users can set their own according to their personal preferences, and have a more comfortable experience, so as to meet everyone's personalized needs as much as possible and occupy more intelligent product market.

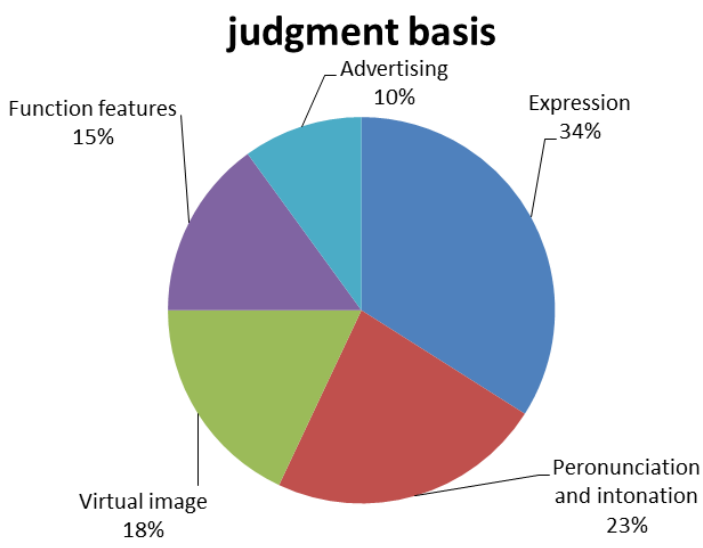

Fig.2 The basis of judging product role

\subsubsection{Pay more attention when buying products}

Respondents pay more attention to the powerful functions of intelligent products when they buy intelligent products. For example, when they buy a sweeping robot, they first pay attention to its cleaning ability, automatic obstacle avoidance function and whether it is suitable for families with children and the elderly, and then they will pay attention to its intelligence and other functions. Therefore, the product should first have perfect functions, and then upgrade the intelligent and emotional interaction. 


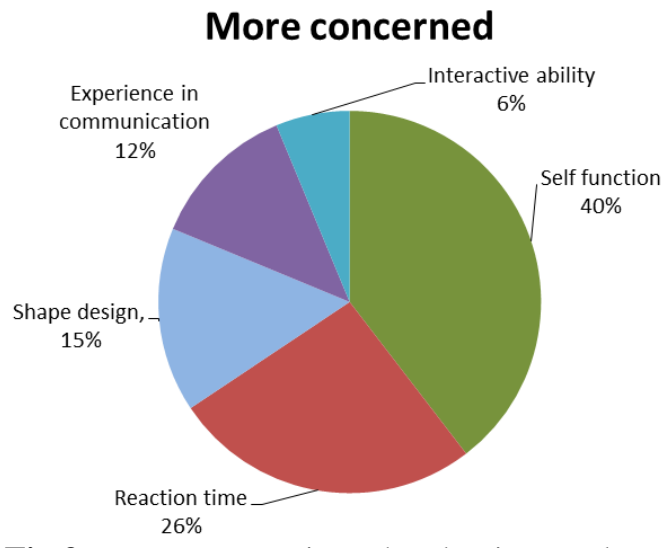

Fig.3 Pay more attention when buying products

\subsection{The following is an analysis of the survey of the focus group forum}

\subsubsection{Response time}

We asked the testers to enter the room one by one, and tested the response time of five kinds of calling intelligent assistants: $600 \mathrm{~ms}, 800 \mathrm{~ms}, 1000 \mathrm{~ms}, 1200 \mathrm{~ms}$ and $1400 \mathrm{~ms}$. The responses show that people are more inclined to receive the response in $800 \mathrm{~ms} \sim 1000 \mathrm{~ms}$. If the response time is less than $800 \mathrm{~ms}$, they will feel interrupted. Moreover, if they respond too eagerly, they will feel monitored all the time. If the response time is longer than $1000 \mathrm{~ms}$, the user will think that the product is too slow to respond to the user's questions or requests in time, which will increase the user's impatience and dissatisfaction, and some test subjects will feel that they are not valued.

\subsubsection{Speed of speech}

We also tested the user's preference for the speech speed of the voice assistant, which is divided into slow speed (7S), medium speed (5S) and fast speed (3S). The data collected show that the test takers prefer medium speed, not only can they hear the content clearly, but also feel comfortable and natural; too fast will have a sense of urgency, cannot hear the content clearly; too slow will not have enough patience to listen to the complete sentence.

\subsubsection{Pronunciation and intonation}

Finally, the test compares the male's high, middle and low bass with the female's high, middle and low bass. We use the computer to simulate different tones and command them to read the same sentence: "I am very glad to serve you. May I help you?" The results show that the subjects prefer mezzo, which can bring them a sense of pleasure and steadiness, and think that the advice or scheme provided is more reliable. For those who have difficult situations to deal with, they will think that such a voice can ease their anxiety and tension, and generate a sense of dependence that they want to solve problems by asking this intelligent assistant next time.

\subsection{Comprehensive analysis of data structure based on questionnaire and focus group}

\subsubsection{Problems in emotional interaction of intelligent products}

At present, most of the intelligent products in the market are not perfect in the function of perceiving users' emotion changes through tone or language, and need to be further upgraded and strengthened. What's more, some intelligent products have slow voice response, mechanized response, no natural humour in the conversation between people, and poor user experience. Besides, the function of interaction with customers should be upgraded, and the user's voice chat interaction experience is poor. Last but not least, personalization functions such as tone, response time and humour adjustment need to be improved.

\subsubsection{Relevant suggestions}

Combined with the statistical results of the questionnaire and the test data analysis of the focus group forum, it is concluded that the product should first have powerful functions, and then improve and upgrade the intelligence and emotional interaction. When users use the intelligent products, they will take them as family or friends. When designing the products, they should pay attention to emotional company and communication from the perspective of similar identity, and strive to bring the same company and existence to the users' family and friends. For intelligent products with voice communication, the response time should be controlled between $800 \mathrm{~ms}$ and $1000 \mathrm{~ms}$. The reply content of the intelligent assistant should first respond to the core requirements and questions put forward by users, and pay attention to that the reply should focus on the target requirements, and tell users clearly and directly what to do and how to do. The use of language is simple and natural, not too complicated or mechanized, with the tone close to daily life and sentence structure, and even according to the user's tone to judge their mood and add some jokes to adjust the atmosphere. The voice tone of voice assistant is the most popular among female middle school voice, which can bring users a pleasant and reliable feeling of using and produce a sense of dependence. 


\section{DISCUSSIONS ABOUT STRATEGIES TO OPTIMIZE THE INTERACTION OF INTELLIGENT PRODUCTS}

\subsection{Personal pronouns}

Professor Clifford NASS and Dr. Scott brave of Stanford University, in their 2005 Book wired for speech, studied whether the voice system should use the personal pronoun "I". The results showed that the design of a recorded voice system using the personal pronoun "I" to reply users would make users feel more relaxed and more like human voice for the speech system using synthetic tone, due to the limitation of sound quality, it is necessary to use "I" with caution [9]. So in the voice system design of intelligent products, the choice and frequency of personal pronouns should be determined according to the main customer groups and product functions, so as to bring the best customer experience.

\subsection{Appearance colour}

For most people, seeing blue makes people feel calm and peaceful; seeing yellow makes people alert and pay attention; seeing green makes people comfortable and relaxed; seeing red makes people warm and excited[10]. Therefore, in product design, we should design different colours according to customers' personal preferences and product design concept.

\subsection{Scene application}

Different scenarios for users to use intelligent products will also bring different use experience. Products should design different scenarios to cooperate with users, so that they can experience a variety of best use feelings in different scenarios. For example, provide a variety of menus and cooking methods in the kitchen, enter the home mode; provide relevant websites or help in work and study, enter the business mode; provide travel routes and accommodation food help when traveling, enter the leisure mode, etc.

\subsection{Emotional interaction}

According to Marx Scheler, "emotion is all human senses, organism, psychology and spirit "It's my feeling." [11] Liu identified the anxiety level of players from the reactions of the autonomic nervous system (ANS), and used it to adapt to the difficulties encountered in table tennis competition; they used similar methods to identify the entertainment of children playing with robots, and let robots change their behaviour to maximize the entertainment [12]. Therefore, the promotion of emotional interaction of intelligent products can greatly improve the user experience and obtain a larger consumer market.

\subsection{Easy to operate}

Any intelligent product should be easy to operate and easy for users to learn to use. For example, the apple watch interface design is simple and convenient, in line with the user's habits; various functional designs make it easy for people to learn, reducing the difficulty of the user's operation. At the same time, the download of app is also very convenient for the learning and use of the elderly and children.

\subsection{Personalized design}

In the view of psychology, personalized, unique or contrary to people's cognitive common sense things are more likely to arouse people's interest and attention. For example, the popular Huawei mobile phone has a simple appearance design, a large screen, few buttons, a unique camera shape and dazzling and colourful appearance colour, which attract consumers' attention and stimulate their purchase desire and behaviour. Therefore, intelligent products should also pay attention to the personalized design of products, such as the tone and volume of voice assistant, response time, product appearance colour, scenario and operation interface.

\subsection{Summary}

Nowadays, the development of intelligent products is very rapid, and various businesses have made a lot of innovations in product design and function. Most of the hard functions of products have been perfect, so the design of emotional interaction must occupy an important position in the future intelligent product design. Therefore, it is very important to optimize the interaction of intelligent products, not only in its own function, appearance design, content improvement, but also in the personification communication with users to make innovation and upgrading.

\section{CONCLUSION}

\subsection{Summary}

In recent years, the emotional interaction of products is a hot topic. With the emergence of various powerful intelligent products on the market, most products mainly focus on the development of their own functions. In order to attract customers' attention and improve user satisfaction, it is necessary to focus on the emotional interaction design of products. Our research found that in addition to the function of the product itself, users prefer to regard intelligent products as friends who can communicate and accompany in their 
lives. This requires more attention to emotional interaction design and intelligence in the design concept and scheme of the product, so as to ensure that the product can accompany and communicate with users to the maximum extent, and make users rely on it. In addition, we should also improve the details in the use of personal pronouns of voice assistant, the diversification of product scene mode, whether it is easy to operate and personalized design, so that users can improve the satisfaction of product use and appearance from all angles.

\subsection{Limitations}

However, there are still limitations in this survey: First, the sample size of the survey is limited; second, most of the respondents are college students, and their ages are generally between 20 and 30 years old; Third, the respondents generally accepted the intelligent products with high learning ability. In the future experiments, we should increase the sample size, age group, occupation category, to make the personnel structure of the research object more comprehensive and scientific, so as to get more comprehensive and convincing experimental data and analysis results.

\section{REFERENCES}

[1] Liao Qinglin, Voice Interaction Design of Smart Home Products Based on Emotional Interaction, Chengdu: Sichuan University, 2019

[2] Norman, D.A, From Fallows Function? Design: why we love( or Hate)everyday things, 2004, New York: cambrage.

[3] LIN Wen-qian. Emotion Recognition and Interaction Application Driven by Physiological Signal[D]. Hang zhou: Zhejiang University, 2019.

[4] Wang Qi, The significance, steps and common problems of writing literature review, Institute of education, Xiamen University, 2010.

[5] Lin Juren, Liu Yu'an, Research methods of social sciences, 2008

[6] GHOSH S, PHERWANI J. Designing of a Natural Voice Assistants for Mobile through User Centered Design Approach[C]//GHOSH S. HumanComputer Interaction: Design and Evaluation. Los Angeles: Springer, 2015: 320-331.

[7] NICULESCU A, DIJK B, NIJHOLT A, et al. Making Social Robots More Attractive: The Effects of Voice Pitch, Humor and Empathy[J]. International Journal of Social Robotics, 2013, 5(2): $171-191$.
[8] LEE B, KWON O, LEE I, et al. Companionship with Smart Home Devices: The Impact of Social Connected ness and Interaction Types on Perceived Social Support and Companionship in Smart Homes $[\mathrm{J}]$. Computers in Human Behavior, 2017(75): 922-934.

[9] Clifford Nass \&Scott Brave, Wired for Speech, MIT Press, 2005

[10] Liu Lin, research on emotional experience design based on interface interaction design

[11] Max Scheler, the founder of modern anthropology, is a famous philosopher of value

[12]LIU C, AGRAWAL P, SARKAR N. Dynamic Difficulty Adjustment in Computer Games Through Real-Time Anxiety-Based Affective Feedback[J]. International Journal of HumanComputer Interaction, 2009, 25(6): 506- 529 